\title{
Neumomediastino secundario a fractura aislada de piso de órbita
}

\author{
Edgard Riquelme M. ${ }^{1}$, Javier Cuéllar G. ${ }^{1}$, Tomás Aitken L. ${ }^{2}$ y Claudio San Martín M. ${ }^{3}$
}

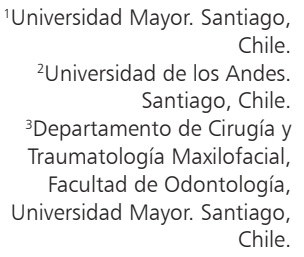

Recibido 2019-11-23 y aceptado 2020-03-03

Correspondencia a: Dr. Edgard Riquelme M. edgard.riquelme.medel@ gmail.com

\section{Pneumomediastinum secondary to isolated fracture of orbit floorx}

Introduction: Pneumomediastinum is defined as the presence of air or gas within the fascial planes of the mediastinum. It is usually a phenomenon secondary to traumatic perforations of the aerodigestive tract. Pneumomediastinum secondary to an orbital fracture is a rare event. And it is related to life-threatening complications such as pneumothorax, pneumopericardium and mediastinitis. Aim: To describe a case of pneumomediastinum secondary to an isolated orbital floor fracture and its medical-surgical management. Clinical case: A 42-year-old female patient who suffers trauma to the facial, cervical and thoracic regions, secondary development of a subcutaneous panfacial emphysema and pneumomediastinum, which resolves successfully. Discussion: Pneumomediastinum following an isolated orbital floor fracture is a very rare event. The air can descend along the fascial spaces to the mediastinum. In this sense, blowing your nose is a risk factor to develop this pathology. Conclusion: After a maxillofacial trauma, emphysema of the deep spaces of the head, neck and even the mediastinum can occur

Key words: pneumomediastinum; orbital fracture; facial fracture; emphysema.

\section{Resumen}

Introducción: El neumomediastino se define como la presencia de aire o gas dentro de los planos fasciales del mediastino. Por lo general, es un fenómeno secundario a perforaciones traumáticas del tracto aerodigestivo. El neumomediastino secundario a una fractura orbitaria es un evento raro. Se asocia a complicaciones potencialmente mortales como el neumotórax, el neumopericardio y la mediastinitis. Objetivo: Describir un caso de neumomediastino secundario a una fractura aislada de piso orbitario y su manejo médico-quirúrgico. Caso clínico: Paciente de sexo femenino de 42 años que sufre traumatismo en regiones facial, cervical y torácica desarrollando secundariamente un enfisema subcutáneo panfacial y un neumomediastino, el cual se resuelve exitosamente. Discusión: El neumomediastino secundario a una fractura aislada de piso orbitario es un evento muy raro. El aire puede descender a lo largo de los espacios fasciales hasta el mediastino. En este sentido, sonarse la nariz es un factor de riesgo para desarrollar esta patología. Conclusión: Ocurrido un trauma maxilofacial puede presentarse enfisemas de espacios profundos de la cabeza, cuello e incluso el mediastino.

Palabras clave: neumomediastino; fractura orbitaria; fractura facial; enfisema.

\section{Introducción}

El neumomediastino se define como la presencia de aire o gas dentro de los planos fasciales del mediastino. Por lo general, es un fenómeno secundario a perforaciones traumáticas del tracto aerodigesti$\mathrm{vo}^{1,2}$. El aire resultante dentro del mediastino puede ascender a través de los planos fasciales causando enfisema subcutáneo. La vía inversa de neumomediastino consecutivo a un trauma maxilofacial es una complicación rara, potencialmente grave y mor- $\mathrm{tal}^{3,4}$. Sonarse la nariz, posteriormente a una trauma facial, aumenta la presión en los senos paranasales, lo que puede provocar ingreso de aire a través de los tejidos blandos circundantes en la cabeza, cuello y en ocasiones hasta el mediastino.

El neumomediastino se asocia más frecuentemente a otro tipo de lesiones más graves que las del trauma maxilofacial, como ruptura esofágica o traqueal, que conllevan a un mayor riesgo de consecuencias potencialmente mortales. Existen cuatro vías de difusión del aire desde las estructuras 
extramediastinales hacia el mediastino propiamente tal: i) a través de los planos fasciales del cuello; ii) a través de una perforación traqueal, bronquial o esofágica en el mediastino; iii) desde el espacio retroperitoneal, a través de los hiatos diafragmáticos y iv) directamente desde los pulmones ${ }^{5}$.

Se describe un peculiar caso de neumomediastino secundario a una fractura aislada de piso de órbita y su resolución médico-quirúrgica.

\section{Caso clínico}

Paciente género femenino de 42 años de edad, en estado etílico, con antecedentes de depresión en tratamiento (lamotrigina), acude a Urgencia de la Clínica Vespucio de Santiago de Chile, tras haber sufrido caída accidental por escaleras recibiendo impactos en región facial, cervical y torácica. Sin pérdida de conciencia. Los signos vitales iniciales mostraron una presión arterial de 114/79 $\mathrm{mmHg}$, una frecuencia cardiaca de 100 latidos/min, una saturación de oxígeno del $98 \%$ y una frecuencia respiratoria de 16 respiraciones/min. El examen físico facial reveló un importante edema periorbitario izquierdo, oclusión palpebral de ojo izquierdo, enfisema de región periorbitaria, geniana, perimandibular y cervical alta de lado izquierdo; presencia de diplopia, disminución de la motilidad ocular e hipoestesia geniana izquierda.

La tomografía computarizada (TC) maxilofacial reveló una fractura tipo Blow Out del piso de la órbita izquierda y un marcado enfisema subcutáneo panfacial (Figura 1). En la TC de cuello se observó una continuidad del enfisema subcutáneo cervicofacial (Figura 2B). En la TC de tórax los principales hallazgos fueron: tráquea y bronquios permeables y de calibre normal, ausencia de adenopatías mediastínicas, vasos mediastínicos de calibre normal sin evidencia de disección, extenso neumomediastino que se acompaña de extenso enfisema que diseca los planos musculares supraclaviculares y subcutáneo, corazón de tamaño conservado sin derrame pericárdico, campos pulmonares bien expandidos sin condensaciones ni nódulos banda atelectásica basales bilaterales, sin derrame pleural y una estructura ósea conservada (Figura 3).

Inicialmente se realizó el ingreso para manejo del enfisema y neumomediastino, se indicó antibioterapia (ceftriaxona) junto con antiinflamatorio/ analgesia endovenosa.

Luego se realizó una evaluación en conjunto con oftalmología, cirugía maxilofacial y cirugía de tórax. Desde el punto de vista oftalmológico, la paciente presentaba previamente astigmatismo y no se encontraron lesiones traumáticas que comprometieran el globo ocular. Por otro lado, se decidió complementar el diagnóstico con una endoscopia/broncoscopia exploratoria para descartar daño a nivel del tracto aerodigestivo, pero tampoco se encontraron lesiones traumáticas.

Finalmente, tras 10 días de hospitalización sin resolución espontánea del enfisema subcutáneo panfacial y del neumomediastino comprobado mediante controles imageneológicos $48 \mathrm{~h}$ previas a la cirugía, se decidió realizar una reconstrucción del piso orbitario con una malla de titanio preformada bajo anestesia general (Figura 4). La TC posoperatoria inmediata a las $24 \mathrm{~h}$ mostró una regresión del enfisema en todos los espacios previamente

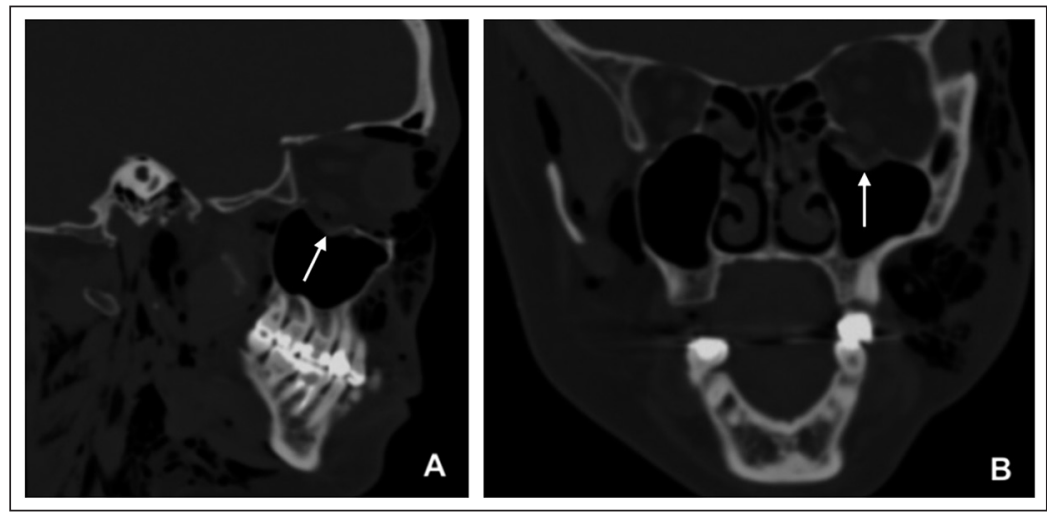

Figura 1. Cortes sagital (A) y coronal (B) de la TC maxilofacial, que revelan una fractura desplazada de piso orbitario izquierdo (flechas) con severo enfisema subcutáneo panfacial.

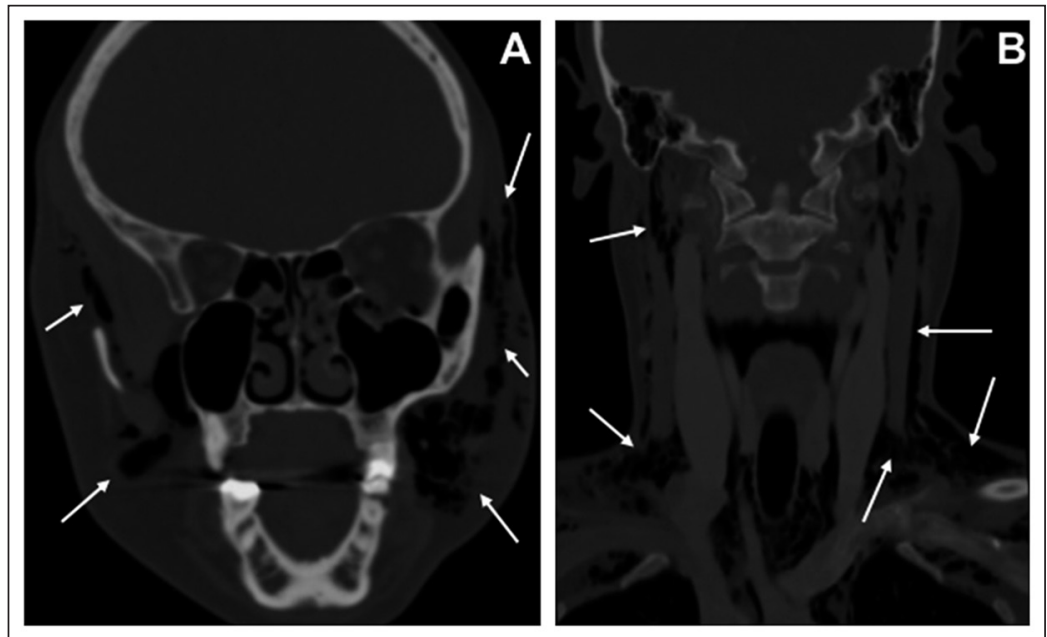

Figura 2. Cortes de la TC que muestran cómo el aire (flechas) ocupa los diferentes espacios de la cabeza de manera ipsi y contralateral a la fractura orbitaria $(\mathbf{A})$, extendiéndose hasta el cuello (B). 

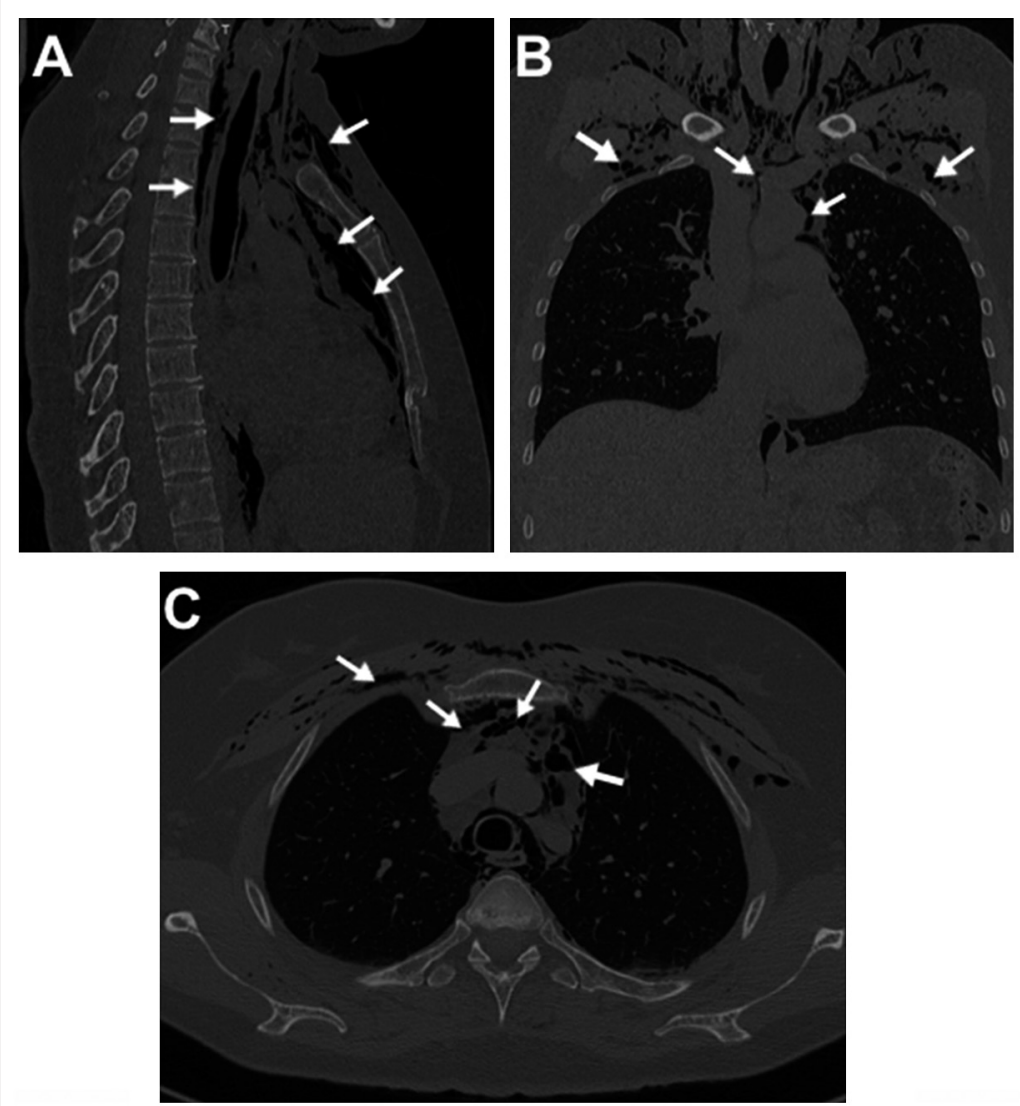

Figura 3. Cortes sagital (A), coronal (B) y axial (C) de la TC de tórax, que muestran como el aire (flechas) comprometen el mediastino superior.

comprometidos y un piso orbitario anatómicamente reconstruido (Figura 5). La paciente fue dada de alta después de $48 \mathrm{~h}$ posterior a la cirugía. En el seguimiento a las 4 semanas, se encontraba completamente asintomática.

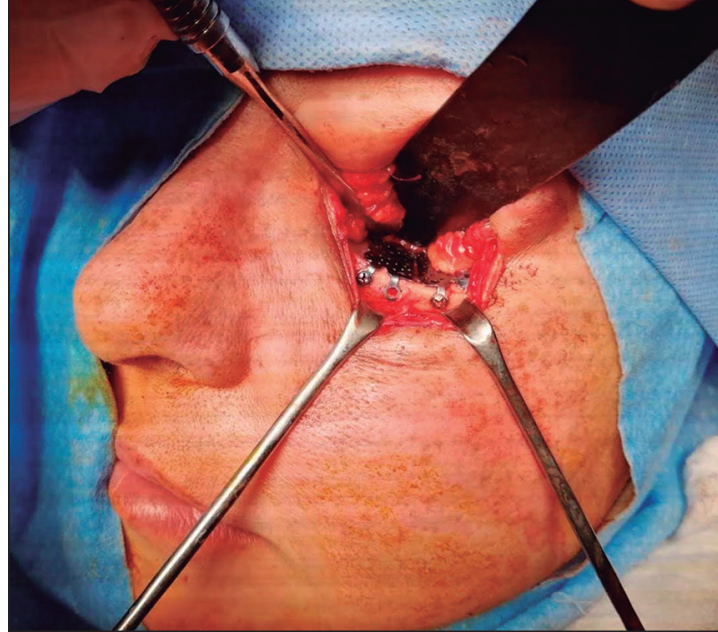

Figura 4. Imagen de la malla de titanio preformada utilizada para la reconstrucción de piso orbitario.

\section{Discusión}

El neumomediastino, enfisema mediastínico o síndrome de Hamman, se define como la presencia de aire o gas dentro de los planos fasciales del mediastino y fue descrito por primera vez en 1827 por Laennec ${ }^{6}$.

El neumomediastino puede ser el resultado de lesiones intratorácicas como la rotura de la tráquea, el bronquio o el esófago debido a un traumatismo torácico cerrado. Una lesión del parénquima pulmonar puede ser otra causa de neumomediastino ${ }^{6,7}$. Estos posibles mecanismos están bien documentados en la literatura, pero el neumomediastino después de un trauma maxilofacial es un evento muy raro. Ocurrido un trauma facial el aire puede descender a lo largo de los espacios fasciales hacia el mediastino. Se ha observado que sonarse la nariz es un factor de
Figura 5. Cortes de la TC posoperatoria inmediata sagital (A) y coronal (B) que muestran la resolución parcial del enfisema y un piso orbitario anatómicamente reconstruido.

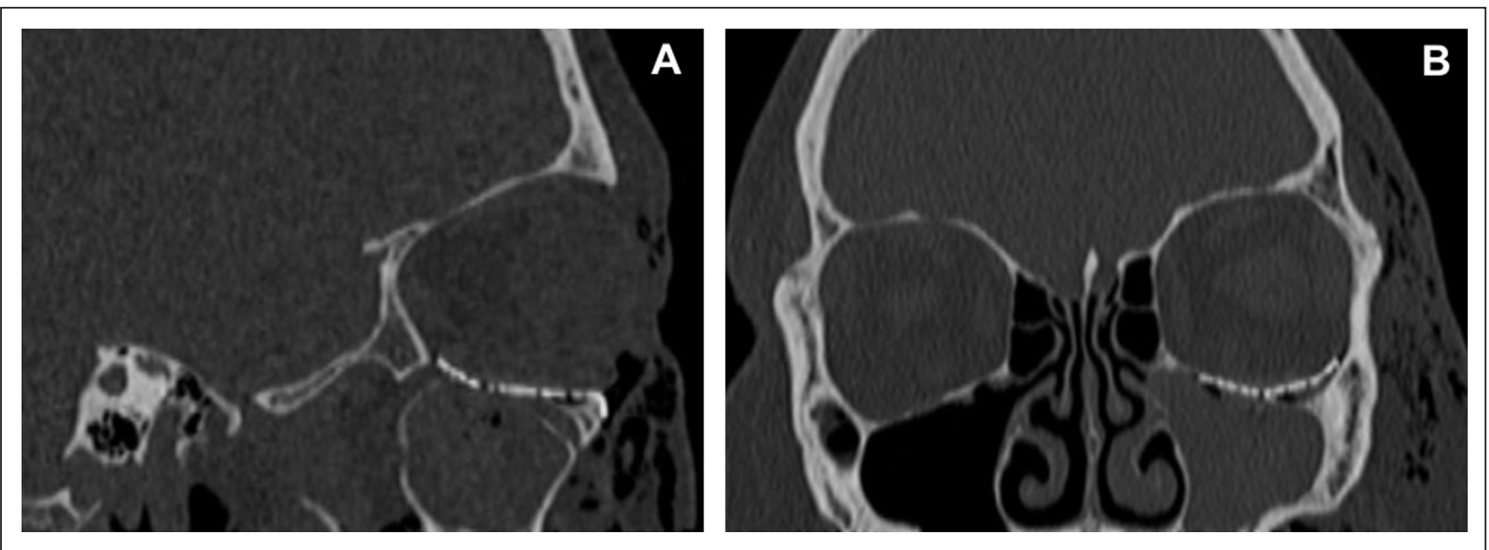


riesgo en la producción de enfisema subcutáneo en fracturas faciales. La espiración forzada repentina, que se acompaña al sonarse la nariz, resulta en una alta presión en las vías respiratorias superiores. Por lo tanto, el aire invade los tejidos adyacentes a través de roturas mucoperiostales relacionadas con las fracturas en los huesos de los senos paranasales. Este es un hallazgo bastante común en las lesiones maxilofaciales, pero en circunstancias excepcionales, el aire puede extenderse al mediastino a través de los espacios submandibular, retrofaríngeo, parafaríngeo, prevertebral y pretraqueal.

Cuando ocurre una fractura orbitaria, una vía de difusión del aire es que pueda ingresar al espacio cigomaticotemporal, extenderse por debajo hacia el espacio bucal y pterigomandibular, para luego alcanzar el espacio parafaríngeo a través del espacio submandibular. El espacio parafaríngeo se comunica posterolateralmente con la vaina carotídea y posteromedialmente con el espacio retrofaríngeo. El espacio retrofaríngeo se extiende hasta la segunda vértebra torácica proporcionando una vía fácil y directa al mediastino posterior. La propagación del aire a través de la fascia visceral del cuello puede alcanzar el mediastino superior anterior ${ }^{8,9}$. Además de este mecanismo, en las fracturas por reventón de la órbita, el aire puede llegar al espacio infratemporal a través de la fisura pterigomaxilar, que se comunica con el espacio pterigopalatino y fisura orbitaria ${ }^{8}$. En nuestro caso, estas dos posibles formas de propagación del aire podrían haber estado involucradas en la ocurrencia del neumomediastino.

En raras ocasiones, el paciente puede tener compresión del corazón, grandes vasos y tráquea como resultado de un aumento de presión asociado al neumomediastino. Puede producirse un retorno venoso reducido con disminución del gasto cardíaco e hipotensión. Los síntomas pueden incluir dolor torácico retroesternal con irradiación en los brazos, el cuello y la espalda; disnea, disfagia y afonía; venas distendidas no pulsátiles del cuello; cianosis y colapso circulatorio. El dolor en el pecho asociado al neumomediastino se agrava con la respiración profunda y por acostarse. El dolor puede diagnosticarse erróneamente como pericarditis, disección de aneurisma aórtico o infarto de miocardio ${ }^{6}$. El signo de Hamman, un sonido similar al crujido, que se escucha sobre el hemitórax izquierdo acentuado durante la sístole y la espiración está presente en aproximadamente en el $50 \%$ de todos los casos de neumomediastino y no debe confundirse con un roce pericárdico ${ }^{6,9,11}$. Este signo no es patognomónico de esta lesión, se describe también en casos de neumotórax del lado izquierdo, dilatación del esófago in- ferior, neumoperitoneo con un diafragma izquierdo alto y dilatación gástrica. La combinación del signo de Hamman y el enfisema subcutáneo permite el diagnóstico de neumomediastino.

Al evaluar a un paciente con trauma maxilofacial que se acompaña de enfisema subcutáneo, el médico debe ser consciente del riesgo latente de un neumomediastino secundario. La atención del médico a esta situación es vital, debido a las complicaciones potencialmente mortales del neumomediastino, como el neumotórax, el neumopericardio y la mediastinitis ${ }^{1,2,8-10}$

El diagnóstico de enfisema mediastínico se realiza mediante la anamnesis del paciente, el examen físico y exámenes complementarios. En cuanto a los exámenes de imágenes, la $\mathrm{TC}$ de tórax es el examen imagenológico de preferencia ${ }^{11}$, aunque las radiografías convencionales de tórax posteroanterior y lateral suelen ser suficientes cuando la TC no esta disponible ${ }^{11,12}$. Procedimientos complementarios como el esofagograma, la esofagoscopia y la broncoscopia ayudan con el diagnóstico porque permiten detectar rupturas del esófago y del árbol traqueobronquial. En nuestro caso, se descartó el traumatismo esofágico cerrado, ya que no existían signos y síntomas como disfagia, odinofagia y fiebre asociados con disnea y dolor torácico. En consecuencia, no sospechamos que el traumatismo esofágico fuese la causa del enfisema subcutáneo y neumomediastino. Por otro lado, si bien nuestro paciente presentaba dolor a nivel cervical y torácico, se descartaron daños de las vías respiratorias, ya que se realizó una broncoscopia donde no se encontraron lesiones. Por lo tanto, se concluyó que la causa del extenso enfisema subcutáneo y el neumomediastino era el trauma orbitario.

Un enfisema cervical extenso y progresivo puede obstruir la vía aérea, lo que pudiese requerir de intubación endotraqueal o traqueotomía. El neumomediastino grave puede ir acompañado de neumotórax en el $50 \%$ de los casos, lo que puede disminuir el retorno venoso y provocar colapso cardiovascular $^{13}$. La mediastinitis es otra posible complicación. Las bacterias potencialmente patógenas del tracto aerodigestivo superior pueden quedar atrapadas en bolsas de aire enfisematosas del cuello y el mediastino ${ }^{14}$.

El manejo del neumomediastino consiste en un monitoreo continuo y en prevenir sus complicaciones asociadas. Se debe administrar un antibiótico intravenoso de amplio espectro como profilaxis de la mediastinitis. Se debe administrar una alta concentración de oxígeno. Esto aumenta el gradiente para la reabsorción en los tejidos, al reducir la presión 
parcial de oxígeno ${ }^{2,6,8}$. Aplicar agujas en la pared torácica y el cuello puede ser útil al liberar aire atrapado hacia el exterior ${ }^{11}$.

Monksfield et al., en su revisión, no documentaron más de 20 casos de neumomediastino secundario a fracturas maxilofaciales y de ellos solo 3 casos estaban relacionados con fracturas aisladas de órbita ${ }^{10}$. Dos fueron fracturas de la pared medial de la órbita y el tercer caso fue una fractura de piso orbitario $^{3,9}$. En todos los casos los pacientes se sonaron la nariz posterior al trauma. Por lo tanto, es importante indicar a los pacientes que no se suenen la nariz después de una lesión maxilofacial. Además, en la misma revisión un solo caso de neumomediastino tras fracturas faciales se trató de forma conservadora, resolviéndose espontáneamente ${ }^{10}$. Solo se ha notificado un caso mortal debido a un neumomediastino asociado con fracturas faciales ${ }^{15}$.

\section{Conclusiones}

El diagnóstico temprano del neumomediastino es vital, ya que su tratamiento depende de su origen y su enfoque va destinado a disminuir sus complicaciones.

Cuando se diagnostica un neumomediastino secundario a fracturas del territorio maxilofacial, siempre se debe descartar posibles lesiones traumáticas del tracto aerodigestivo.

Siempre se debe advertir a los pacientes con trauma maxilofacial sobre la importancia de evitar sonarse la nariz, para evitar enfisemas en espacios profundos de la cabeza, cuello y eventualmente el mediastino.

\section{Responsabilidades éticas}

Protección de personas y animales. Los autores declaran que para esta investigación no se han realizado experimentos en seres humanos ni en animales

Confidencialidad de los datos. Los autores declaran que en este artículo no aparecen datos de pacientes.

Conflictos de interés: no hay

\section{Bibliografía}

1. Millard CE. Pneumomediastinum. Dis Chest. 1969;56:297-300. DOI: 10.1378/ chest.56.4.297.

2. Munsell WP. Pneumomediastinum. JAMA 1967;202:689-93. DOI: 10.1001/ jama.202.8.689.

3. Habal MB, Beart R, Murray JE. Mediastinal emphysema secondary to a fracture of the orbital floor. Am J Surg. 1972;123:606-8. DOI: 10.1016/00029610(72)90232-2.

4. Abdelrahman H, Shunni A, El-Menyar A, Ajaj A, Afifi I, Zarour A, et al. Mediastinal emphysema following fracture of the orbital floor. J Surg Case Rep. 2014;2014: rju032. DOI: 10.1093/jscr/rju032.

5. Hollinshead WH. Fascia and fascial spaces of the head and neck. In: English GM, editor. Anatomy for surgeons. The Head and Neck, 2nd ed. New York: Harper \& Row; 1982. p. 306-30.

6. Minton G, Tu HK. Pneumomediastinum, pneumothorax, and cervical emphysema following mandibular fractures. Oral Surg Oral Med Oral Pathol. 1984;57: 490-3. DOI: 10.1016/00304220(84)90305-0.

7. López-Pelaez MF, Roldan J, Mateo S. Cervical emphysema, pneumomediastinum, and pneumothorax following self-induced oral injury: report of four cases and review of the literature. Chest 2001;120:306-9. DOI: 10.1378/ chest.120.1.306.

8. Henry CH, Hills EC. Traumatic emphysema of head, neck and mediastinum associated with maxillofacial trauma: case report and review. J Oral Maxillofac Surg. 1989;47:876-82. DOI: 10.1016/s02782391(89)80053-9.

9. Roccia F, Griffa A, Berardino M. Severe subcutaneous emphysema and pneumomediastinum associated with minor maxillofacial trauma. J Craniofacial Surg. 2003;14:880-3.
10. Monksfield $\mathrm{P}$, Whiteside $\mathrm{O}$, Jaffé S, Steventon N, Milford C. Pneumomediastinum, an unusual complication of facial trauma. Ear Nose Throat J. 2005;84:298-301.

11. Zylak CM, Standen JR, Barnes GR, Zylak CJ. Pneumomediastinum revisited. Radiographics. 2000;20:1043-57. DOI: 10.1148/ radiographics.20.4.g00j1131043.

12. Hamman L. Mediastinal emphysema. JAMA 1945;1:128-33.

13. Kirchner JA. Cervical mediastinal emphysema. Arch Otolaryngol. 1980;06:368-75. DOI: 10.1001/ archotol.1980.00790300056012.

14. Stockdale CR. Surgical emphysema of the face following a middle third fracture. Oral Surg. 1958;1:135. DOI: 10.1016/00304220(58)90053-7.

15. Gouda HS, Mestri SC. Mediastinal emphysema due to an isolated facial trauma: a case report. Med Sci Law 2008;48:178-80. 